\title{
Cucurbit[7]uril-Capped Hybrid Conjugated Oligomer-Gold Nanoparticles for Combined Photodynamic-Photothermal Therapy and Cellular Imaging
}

\author{
Melis Özkan, Seyed Ehsan Hadi, İlknur Tunç, Yakup Midilli, Bülend Ortaç, and Dönüs Tuncel*
}

Cite This: ACS Appl. Polym. Mater. 2020, 2, 3840-3849

Read Online

ACCESS | Llll Metrics \& More | 国 Article Recommendations | st Supporting Information

ABSTRACT: Herein, hybrid nanoparticles composed of a redemitting conjugated oligomer (COL) and gold nanoparticles (AuNPs) were prepared through a one-pot synthetic method in which the oligomer acts as a reducing agent as well as a matrix to wrap the newly formed Au nanoparticles. These hybrid nanoparticles (COL-Au-NPs) exhibited photodynamic and photothermal activity against both Gram-positive and Gram-negative bacterial strains. They were also proven to possess high photostability and thermal reversibility. Dark cytotoxicity of COL-Au-NPs toward pathogens and mammalian breast cancer cells (MCF-7) reduced significantly upon complexation with cucurbit[7]uril while preserving their light-induced cytotoxic activity when irradiated with a $915 \mathrm{~nm}$ laser for photothermal therapy and white light for
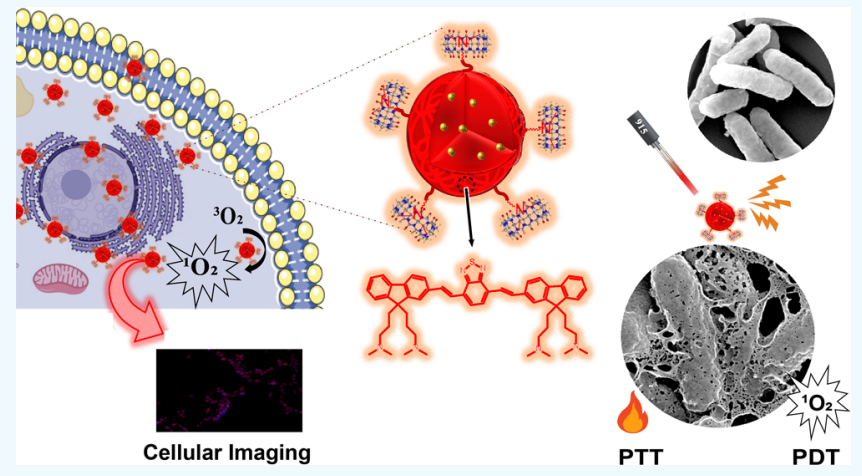
photodynamic therapy, respectively. Furthermore, these nanoparticles have cellular imaging capability because of their intrinsic fluorescence characteristics and can be used in image-guided therapy.

KEYWORDS: cucurbituril, conjugated oligomer, gold nanoparticles, cellular imaging, combined photodynamic-photothermal antibacterial therapy, cancer therapy

\section{INTRODUCTION}

Over the past few decades, alternative therapeutic strategies have been sought after for the treatment of infectious diseases and cancer. In this regard, nanomaterial-based systems are getting increasing attention as more than one functionalities and properties can be combined in one platform to fight with infectious diseases and cancer more efficiently. ${ }^{1-4}$ Recently, therapeutic strategies using light including photodynamic and photothermal therapies (PDT and PTT, respectively) have started to emerge, especially because of serious concerns related to the rapid evolution of antidrug-resistant pathogens. ${ }^{5,6}$ They are also clinically approved because they cause minimal invasiveness and minimal side effects in comparison with the other conventional therapies. ${ }^{7-9}$

For PDT, three important components are essential; they are a photosensitizer, light, and molecular oxygen. ${ }^{10,11}$ In PDT, an ideal photosensitizer should be water soluble, stable, and have a high singlet oxygen generation ability. It should also not display dark toxicity. When an appropriate photosensitizer is excited by a specific wavelength of light, reactive oxygen species (ROS) including singlet oxygens are generated from the molecular oxygens through electron transfer or energy transfer. ROS interact with many parts of cellular components through oxidative reactions; this, in turn, causes an inhibition in the activities of the cells including enzymes, lipids, proteins, and DNA leading to cell death. ROS can destroy the bacteria by damaging the cellular membranes resulting in the leakage of cellular contents that cause an inactivation of enzymes and shutting down of their membrane transport systems. ${ }^{12}$

On the other hand, PTT relies on light-induced heat generation by a photosensitizer absorbing in the near-infrared (NIR) region. When the photosensitizer is excited, it generates heat causing hyperthermia to destroy tumor cells or pathogens. ${ }^{13-17}$ The use of nanomaterial-based systems allows the integration of suitable photosensitizers for both PDT and PTT in one platform to be used in multimodal synergistic therapy strategies, which enhance therapeutic outputs of individual therapies. ${ }^{16-19}$

In theory, hyperthermia caused by a photothermal conversion accelerates an intratumoral blood flow leading to an increase in the oxygen demand for a more efficient photodynamic effect. $^{20,21}$ Another advantage of the synergistic

Received: May 22, 2020

Accepted: July 7, 2020

Published: July 7, 2020 
PDT and PTT is that the temperature elevation by a photothermal effect could promote an enhanced permeability and an efficient cellular uptake of injected photosensitizers. $^{22-24}$

Usually small organic dye-based photosensitizers for PDT are combined with gold and silver nanoparticles that display a considerable localized surface plasmon resonance in the NIR region, and this allows NIR light energy to be converted to thermal energy in high yield for PTT. ${ }^{25}$ These small organic molecule-based photosensitizers are generally incorporated into $\mathrm{Au}$ or $\mathrm{Ag}$ nanoparticles through noncovalent interactions. This approach presents some problems such as a low photostability and the detachment of these dyes from the metal nanoparticles, which, in turn, might cause a decrease in the efficiency.

Conjugated polymer and oligomer nanoparticles have been employed as photosensitizers by utilizing their light-harvesting capabilities, tunable properties, and facile functionalization. $^{26-29}$ Although in the literature there are examples on the hybrid nanoparticles of conjugated polymers with $\mathrm{Au}$ or $\mathrm{Ag}$ nanaoparticles, ${ }^{30-32}$ these usually involve the addition of preformed metal nanoparticles to the conjugated polymers to form core-shell or raspberry-type nanoparticles, and also their combined PDT/PTT applications have not been reported. Recently, we developed a method in which hybrid nanoparticles are formed in one pot by in situ formation of $\mathrm{Ag}$ nanoparticles in the presence of a conjugated oligomer $(\mathrm{COL}){ }^{33}$ In this method, the COL acted as a reducing agent and a matrix that wrapped the newly formed $\mathrm{Ag}$ nanoparticles and also stabilized through interactions with the binding sites present on the matrix. In this work, we adopted our recently developed method for the synthesis of Aucontaining hybrid nanoparticles and demonstrated that this method can be applied to the synthesis of other metal nanoparticles and employed in both PDT and PTT as well as for the broad-spectrum antibacterial combined PDT/PTT effect on bacteria. To the best of our knowledge, this is a first example on the application of these kinds of hybrid nanoparticles in combined PDT/PTT, photodynamic cancer therapy, and cellular imaging. We also ensured that our hybrid nanoparticles (COL-Au-NPs) do not display dark toxicity, which is one of the important criteria to be an ideal photosensitizer for PDT and PTT. For this purpose, we adopted our previously reported method in which amine groups of conjugated oligomer nanoparticles (COL-NPs) that cause toxicity were capped with cucurbit[7] uril (CB7). ${ }^{34}$ CB7 is a macrocycle with a hydrophobic cavity and two hydrophilic portals containing carbonyl groups and known to have a high affinity toward amine groups. CB7 among other CB homologs is the most water-soluble one. ${ }^{35,36}$ Because of these features, CB7 has become highly attractive for the regulation of the cytotoxicity of nanomaterials and drugs. ${ }^{37-40}$

\section{MATERIALS AND METHODS}

2.1. Experimental Section. A red-emitting COL, namely, $3,3^{\prime}, 3^{\prime \prime}, 3^{\prime \prime \prime}-\left\{\left[\left(1 E, 1^{\prime} E\right)-2,1,3\right.\right.$-benzothiadiazole-4,7-diylbis(ethene-2,1diyl)]-bis( $9 H$-fluorene-9,9,2-triyl) $\}$-tetrakis $(N, N$-dimethylpropan-1amine), was synthesized according to the literature procedure. ${ }^{34,41}$ ROS generation efficiency was determined using dichlorofluorescein diacetate (DCFH-DA) assay. ${ }^{42}$ Preparation of Escherichia coli (E. coli) and Bacillus subtilis (B. subtilis) suspensions, determination of minimum inhibitory concentration (MIC), photodynamic inactivation of bacteria, and performing scanning electron microscopy (SEM) imaging, $\zeta$-potential measurements, and cytotoxicity assays of nanoparticles were carried out using similar procedures reported in our previous publications, ${ }^{43-45}$ and details are also given in the Supporting Infomation.

2.2. Preparation of $\mathrm{COL}$ and $\mathrm{COL}-\mathrm{Au}$ (COL/Au, 2:1) Nanoparticles. Synthesis and characterization of the oligomer were reported in our previous publications in detail. ${ }^{34,41}$ To prepare the COL, $5 \mathrm{mg}(5.8 \mu \mathrm{mol})$ of oligomer was dissolved in $5 \mathrm{~mL}$ of THF. Oligomer $(2.35 \mu \mathrm{mol})$ solution $(2 \mathrm{~mL})$ was added to $15 \mathrm{~mL}$ of Milli-Q water while sonicating for $30 \mathrm{~min}$ at medium intensity. THF was evaporated using a rotary evaporator under reduced pressure. Hybrid COL-Au nanoparticles were obtained by injecting $2 \mathrm{~mL}$ of THF solution of oligomer $(2.35 \mu \mathrm{mol})$ into $20 \mathrm{~mL}$ aqueous solution of $\mathrm{AuCl}_{3}(1.18 \mu \mathrm{mol})$ dropwise. The mixture was sonicated for 30 min at $25{ }^{\circ} \mathrm{C}$ and then THF was evaporated under reduced pressure to obtain a stable hybrid core-shell COL-Au nanoparticle dispersion in water, which was further poured into a regenerated cellulose membrane (MW cut-off $12 \mathrm{kDa}$ ) and dialyzed against water for $6 \mathrm{~h}$ to remove unreacted $\mathrm{Au}$ ions.

2.3. Capping COL-NPs and COL-Au-NPs by CB7. An aqueous solution of $\mathrm{CB} 7$ was added to the prepared COL-NPs and conjugated oligomer-gold nanoparticles (COL-Au-NPs) by keeping the concentration ratio at 1:4 (oligomer/CB7) and mixed gently. Then mixtures were poured into a regenerated cellulose membrane (MW cut-off 12 $\mathrm{kDa}$ ) and dialyzed against water for $6 \mathrm{~h}$ to remove excess CB7.

2.4. Measurement of Photothermal Conversion Efficiency and Thermal Reversibility. The photothermal properties of COL, CB7@COL,COL-Au, and CB7@COL-Au nanoparticles were analyzed by measuring the temperature increment upon NIR laser irradiation. Solutions $(1 \mathrm{~mL})$ of each sample were placed in an Eppendorf tube and exposed to NIR irradiation $(915 \mathrm{~nm}$ ) for $10 \mathrm{~min}$. After the irradiation period, to observe thermal reversibility, the laser was turned off and the temperature decrease was measured for $10 \mathrm{~min}$ using an infrared (IR) camera. To examine concentration- and laser intensity-dependence of photothermal performance, the same protocol was followed by varying nanoparticle concentrations and laser density.

2.5. Antibacterial PDT and PTT Efficacy Analyzed by Agar Disk Diffusion Assay. The antibacterial PDT and PTT efficacies of nanoparticles toward E. coli and B. subtilis bacteria were analyzed by agar disk diffusion assay. Both $E$. coli and $B$. subtilis strains were inoculated in Luria-Bertani (LB) and grown $\left(37^{\circ} \mathrm{C}, 200 \mathrm{rpm}, 14 \mathrm{~h}\right)$. Bacterial cultures were diluted 100 -fold with fresh LB and grown to the mid-log phase $\left(\mathrm{OD}_{600} \sim 0.5-0.6\right)$. From each strain, $150 \mu \mathrm{L}$ of cultures were spread evenly throughout the agar plates. A volume of $10 \mu \mathrm{L}$ from each nanoparticle and test antibiotic (ampicillin) were placed on circular filter papers ( $5 \mathrm{~mm}$ in diameter). The UV sterilized dry disks were placed on the agar plates at room temperature. Agar plates were incubated for $14 \mathrm{~h}$ at $37^{\circ} \mathrm{C}$. The antibacterial activities of nanoparticles were visualized by measuring the radii of the inhibition zones after $3 \mathrm{~min}$ of white light exposure $\left(22 \mathrm{~mW} / \mathrm{cm}^{2}\right)$ for PDT, 3 min laser exposure $\left(915 \mathrm{~nm}, 7 \mathrm{~W} / \mathrm{cm}^{2}\right)$ for PTT, and consecutive white light and laser exposure for combined PDT/PTT evaluations.

2.6. Preparation of Cell Fixative Solution. A volume of $20 \mathrm{~mL}$ of $1 \mathrm{X}$ PBS was heated to $60^{\circ} \mathrm{C}$ while stirring in a round-bottom flask, and $1 \mathrm{~g}$ of paraformaldehyde powder was transferred to the flask. $\mathrm{NaOH}(1 \mathrm{~N})$ was added dropwise until the solution became clear. Once paraformaldehyde was completely dissolved, the solution was cooled to room temperature and filtered. The total volume was adjusted to $25 \mathrm{~mL}$ by adding $1 \mathrm{X}$ PBS. The dilute $\mathrm{HCl}$ solution was added to set the final $\mathrm{pH}$ to 6.9 . The resulting solution was stored at 4 ${ }^{\circ} \mathrm{C}$ in the dark.

2.7. CLSM Experiments. MCF-7 cells were cultured on six-well tissue culture plates at a density of $5 \times 10^{5}$ per well in DMEM as described above. After $24 \mathrm{~h}$, cells were treated with $1 \mu \mathrm{M}$ of each nanoparticle group for comparison with the no-treatment control group. After $48 \mathrm{~h}$, the light group was exposed to white light for 20 min, while the dark group was kept in an incubator. Next day, the medium was aspirated, and cells were rinsed with PBS three times (5 min each) and fixed for $10 \mathrm{~min}$ in $4 \%$ paraformaldehyde. The fixative was aspirated, and cells were rinsed with PBS three times $(5 \mathrm{~min}$ 
each) Then, the cells were permeabilized in $0.1 \%$ Triton X-100 for 5 min. After aspirating the Triton, cells were rinsed with PBS three times $(5 \mathrm{~min}$ each). DAPI stock solution $(1 \mathrm{mg} / \mathrm{mL})$ was diluted 1000 -fold in PBS, and cells were incubated with diluted DAPI solution at room temperature for $5 \mathrm{~min}$. DAPI was aspirated, and cells were rinsed with PBS three times.

\section{RESULTS AND DISCUSSION}

3.1. Synthesis and Characterization of Nanoparticles. Scheme 1 shows a schematic view of the preparation of COL-

Scheme 1. Schematic Overview of the Preparation of (a) COL-NPs and (b) COL-Au-NPs and Complexation of Amine Residues of NPs with CB7, (c) CB7@COL-NP and (d) CB7@COL-Au-NPs

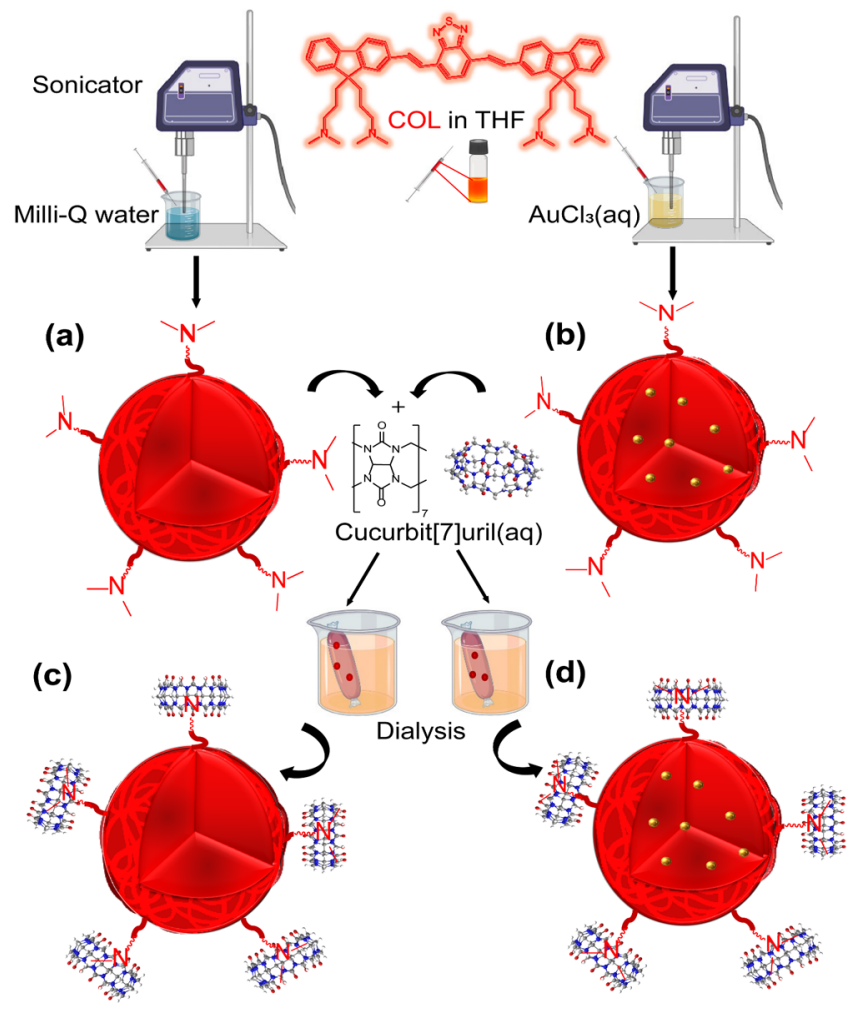

NPs and COL-Au-NPs and complexation of amine residues of NPs with CB7. Synthesis of a benzothiadiazole- and fluorenebased COL was reported in our previous publications. ${ }^{34,41}$ Nanoparticles were prepared using a one-pot method employed previously for the synthesis of a hybrid COL containing silver nanoparticles. ${ }^{33}$ Briefly, the COL was dissolved in tetrahydrofuran (THF) and added dropwise to water and aqueous $\mathrm{AuCl}_{3}$ solution under sonication to form COL-NPs and COL-Au-NPs, respectively. In this method, metal nanoparticles are formed in situ without the necessity of an extra reducing agent as the tertiary amine pendant groupcontaining COL acts as a reducing agent and also as ligands to stabilize the newly formed metal nanoparticles. ${ }^{33}$ We have tried different ratios of COL to gold ion concentration and found 2:1, COL:Au-NP, to be an optimum ratio. Raspberry-type design $^{33}$ for the fabrication of hybrid nanoparticles was also tried. In this design, NPs are prepared by first forming COLNPs and then treated with gold ions to allow the formation of $\mathrm{Au}$ NPs on the COL-NP. However, NPs obtained through this design yield rather unstable particles, which undergo agglomeration over time. Therefore, in our further studies we prepared the hybrid nanoparticles with the optimum ratio (2:1, $\mathrm{COL} / \mathrm{Au}$ ) and $\mathrm{Au}-\mathrm{NPs}$ wrapped by COL design.

Dynamic light scattering (DLS) measurements were performed to determine the sizes of nanoparticles synthesized under the abovementioned conditions, and the average hydrodynamic diameter was measured to be around $120 \mathrm{~nm}$ for COL-NPs and CB7@COL-NPs, while it decreased to 80 nm for COL-Au-NPs and CB7@COL-Au-NPs (Supporting Information, Figure S1). The $\zeta$-potential measurements revealed that COL-NPs and COL-Au-NPs have zeta potentials of $+50 \mathrm{mV}$, and the amine functional groups in the pendant chain of the oligomer account for these positive values of $\zeta$ potentials, which demonstrates the formation of a stable nanoparticle dispersion enabled by repulsion between the nanoparticles. When they are capped with CB7, their sizes remain the same, but their $\zeta$-potential values decrease to around $+25 \mathrm{mV}$ because of the presence of partially negatively charged carbonyl units of CB7. In Table S1, the average zeta size, polydispersity index (PDI), and $\zeta$-potential of nanoparticles are given.

Their sizes and morphologies were further investigated by taking images using transmission electron microscopy (TEM) and a SEM (Figure 1). Micrograms show that COL-Au-NPs are spherical with smooth surfaces and Au NPs are distributed throughout the COL-NPs. The compositional analysis of COL-Au-NPs was performed by running energy dispersive Xray spectroscopy (EDX) as shown in Figure 1c. Au signals were observed in the specified reduced inner area of hybrid nanoparticles in the EDX spectrum indicating that Au-NPs formed successfully.

The X-ray diffraction (XRD) patterns of the COL-Au nanoparticles were acquired by drop casting their aqueous dispersions on microscope slides (Figure S5). While the COL is amorphous as can be seen from its XRD spectrum, $2 \theta$ peaks at 38.1, 44.3, 64.5, and 77.7 were observed in the XRD pattern of COL-Au-NPs corresponding to the reflections from the (111), (200), (220), and (311) planes for Au-NPs wrapped by the COL. X-ray photoelectron spectroscopy (XPS) was also used to characterize the COL-Au-NPs. Figure S6a represents the XPS survey scan of the COL-Au-NP sample. As Au-NPs are wrapped by the COL, $\mathrm{Au}$ signals (Figure S6b) are much weaker than the signals of $\mathrm{C}, \mathrm{N}$, and $\mathrm{S}$, suggesting that the $\mathrm{COL}$ accumulating on the Au nanoparticles causes attenuation in the Au XPS signal; in addition, the Au signal has a low crosssectional value.

3.2. Photophysical and Photothermal Properties of Nanoparticles. Photophysical properties of COL-NP and COL-Au-NPs were investigated by UV-vis-NIR absorption spectroscopy, dynamic spectroscopy, and time-resolved fluorescence (TRF) spectroscopy. While the UV-vis-NIR spectrum of COL-NPs in water shows two major peaks at around 350 and $457 \mathrm{~nm}$, the peak at $457 \mathrm{~nm}$ red-shifted $12 \mathrm{~nm}$ for the hybrid nanoparticles of COL-Au and became relatively sharper compared to that for COL-NPs. This observation can be explained by the contribution of an additional plasmonic band of $\mathrm{Au}$ nanoparticles besides the absorption peak of the COL (Figure S3).

The average lifetime for the samples was calculated and reported as both the intensity-averaged and amplitudeaveraged lifetimes (Table S2) by collecting their photoluminescence $(\mathrm{PL})$ decay curves at their PL emission peak. For both the aqueous dispersions of COL-NPs and COL-Au-NPs, 

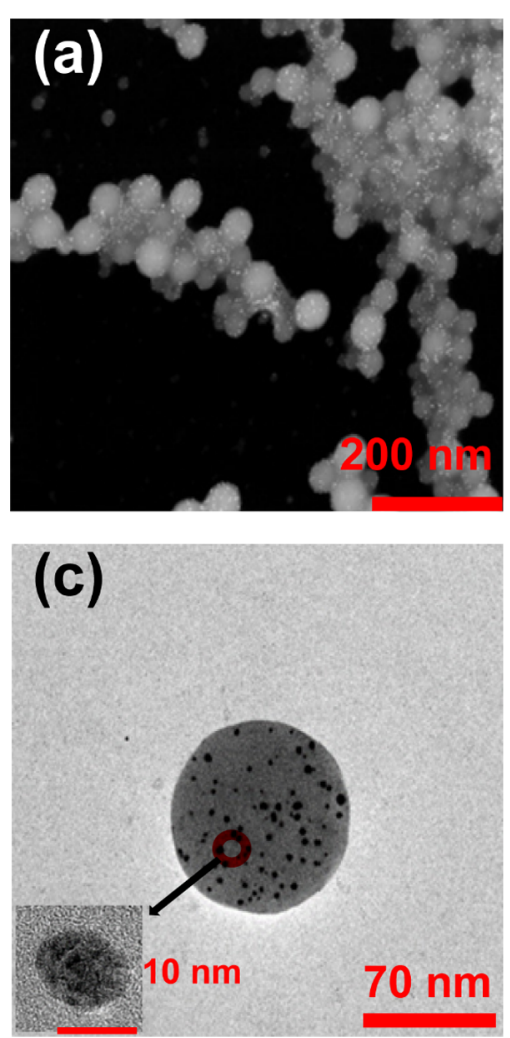
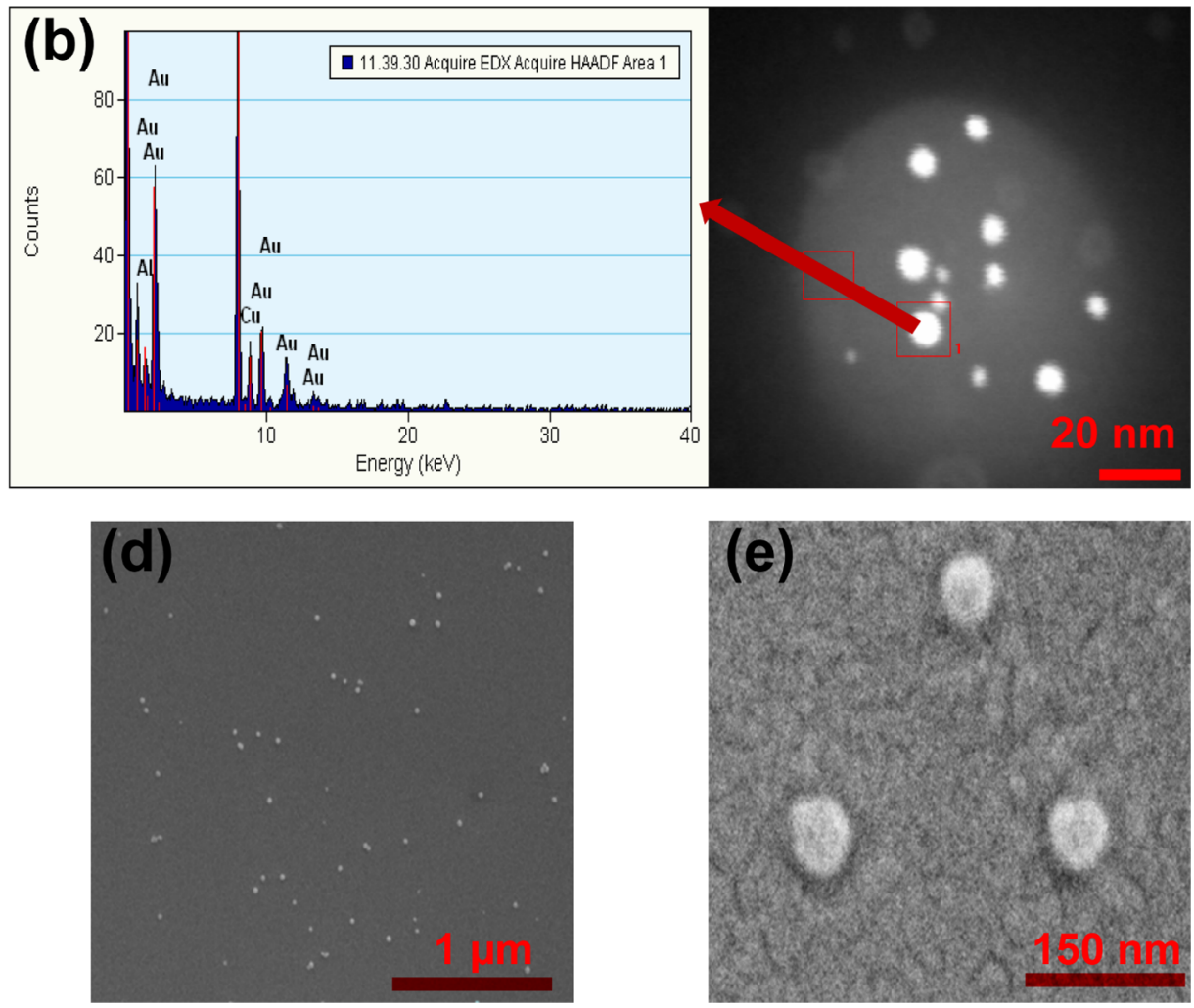

Figure 1. Morphological characterization of COL-Au-NPs through electron microscopy: (a) HAADF-STEM image, (b) EDX analysis, (c) TEM image, and (d) and (e) SEM images.

the fluorescence lifetime and quantum yield significantly decreased compared to the COL solution in THF because of folding of COL chains in water, which cause them to be in close contact as well as a significant increase in their nonradiative channels. ${ }^{27}$

The ROS generation efficiency of COL-NPs and COL-AuNPs under white light exposure $\left(1 \mathrm{~mW} / \mathrm{cm}^{2}\right)$ was investigated using the DCFH-DA assay. ${ }^{42,46}$ When 2,7-dichlorofluorescein $(\mathrm{DCFH})$ was irradiated, a very weak emission peak at $524 \mathrm{~nm}$ appeared because of auto-oxidation of DCFH to DCF. With adding the COL-Au or COL nanoparticles to the solution and starting the illumination, the peak intensity at $524 \mathrm{~nm}$ started increasing significantly and continued to rise gradually over the illumination time (Figure S7). These findings imply that both COL-NPs and COL-Au-NPs can be considered as good photosensitizers with high ROS generation ability even under the low flux of white light. When the ROS generation capacities of COL-NPs and COL-Au-NPs were compared, the ROS generation ability of COL-Au-NPs was observed to be higher. This can be attributed to the metal-enhanced ROS

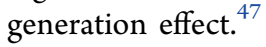

Encouraged by this result, we next investigated the photothermal properties of our hybrid nanoparticles, although we have not observed a significant plasmonic absorption peak for COL-Au-NPs in the NIR region of the spectrum. For this purpose, aqueous dispersions of COL-NP, CB7@COL-NP, COL-Au-NPs, and CB7@COL-Au-NP were irradiated with a NIR laser of $915 \mathrm{~nm}$ wavelength by varying intensities (fluences between 1 and $7 \mathrm{~W} / \mathrm{cm}^{2}$ ) and concentrations (1$10 \mu \mathrm{M}$ ), and the heat release causing an increase in the temperature was recorded using a thermal camera. The results are presented in Figure 2 and Figure S8. Optimum conditions were selected as $10 \mu \mathrm{M}$ with a flux of $7 \mathrm{~W} / \mathrm{cm}^{2}$ and a wavelength of $915 \mathrm{~nm}$ as a maximum heat release was observed under these conditions. Under these conditions, the photothermal conversion efficiency of COL-Au-NPs was calculated as $15 \%$ using a literature method, ${ }^{48}$ and the calculations are shown in the Supporting Information. Laser intensity may seem higher than the ones reported in the literature, but the concentration of nanoparticles is quite low $(10 \mu \mathrm{M}=8.6 \mu \mathrm{g} /$ $\mathrm{mL}$ ). Instead of increasing concentration, laser density was increased to be more suitable for bacterial assays. The photothermal response of nanoparticles over time is shown in Figure 2a. CB7-capped COL-Au-NPs exhibit almost the same response as naive COL-Au-NPs. The rapid temperature rises of COL-Au-NPs and CB7@COL-Au-NP over $45{ }^{\circ} \mathrm{C}$ within $5 \mathrm{~min}$ and $52{ }^{\circ} \mathrm{C}$ at the end of the irradiation period even at low concentration verify their photothermal properties. After $10 \mathrm{~min}$, when the laser was shut off, the temperature decreased almost to the initial temperature showing the reversibility of the process. NIR thermal images are provided in Figure 2 b. After five sequential ON/OFF irradiation cycles (5 min each), ${ }^{15}$ COL-Au-NPs have maintained their photothermal heating performance (Figure 2c); this is one of the important requirements to be considered as an ideal photothermal agent. In addition to their high photothermal stability, COL-Au-NPs display a concentration-dependent temperature increase (Figure $2 \mathrm{~d}$ ) at a constant laser density. It is also observed that temperature increases at a fixed concentration by varying the laser density (Figure S8).

3.3. Photodynamic Effects of Nanoparticles on Bacteria. After finding out that COL-Au-NPs are a good photosensitizer with an ability to generate singlet oxygen, their light-induced biological activities were investigated on bacteria 

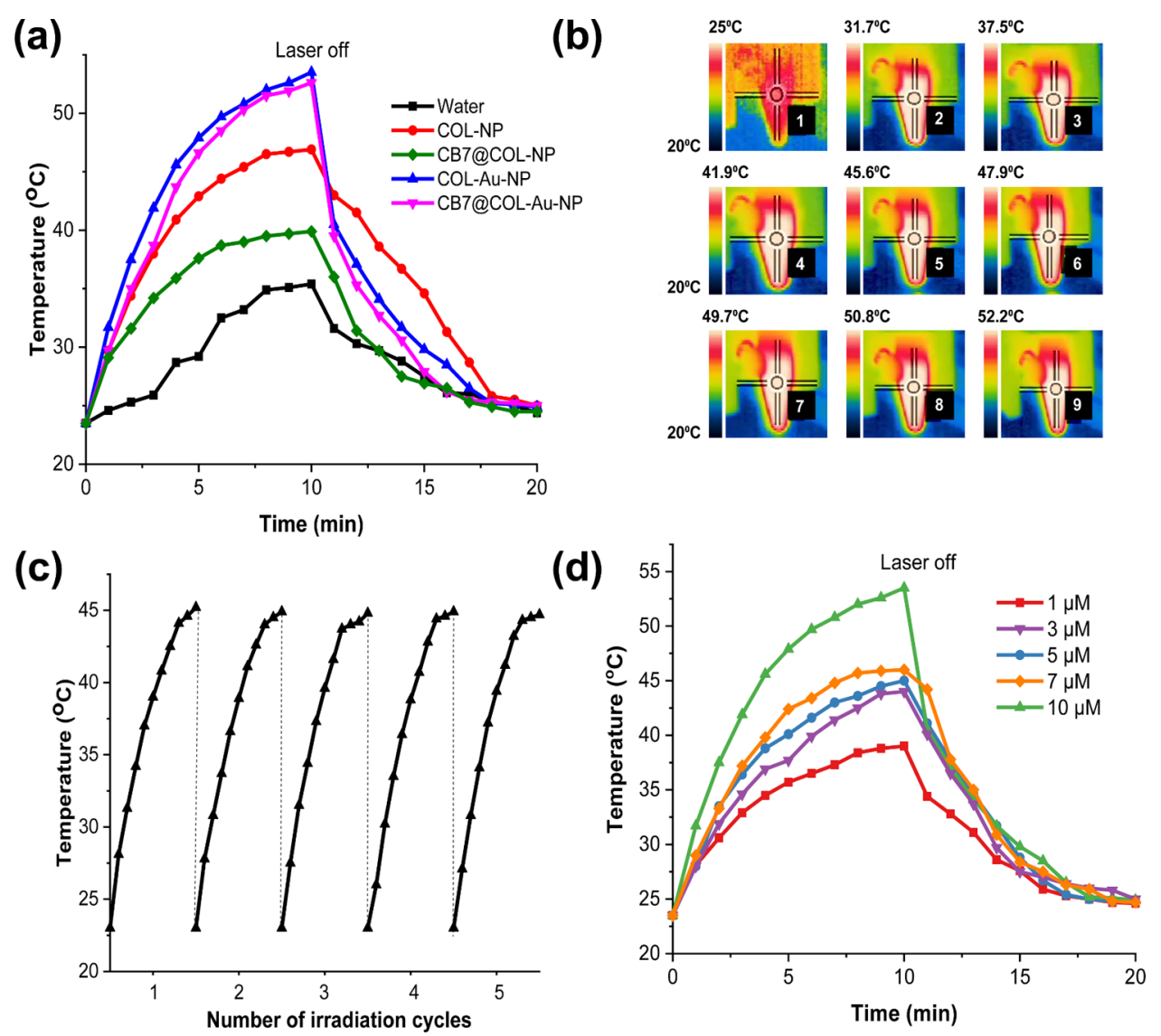

Figure 2. (a) Photothermal response of COL-NPs, CB7@COL-NP, COL-Au-NPs, and CB7@COL-Au-NPs (COL concentration is 10 $\mu \mathrm{M}$ in each) under $915 \mathrm{~nm}$ ON/OFF laser irradiation $\left(7 \mathrm{~W} / \mathrm{cm}^{2}\right)$. After $10 \mathrm{~min}$ of continuous irradiation, the laser was shut off, and Milli-Q water was measured as a blank. (b) IR thermal images of COL-Au-NPs $(10 \mu \mathrm{M})$ for $9 \mathrm{~min}$, and the initial temperature was $23.5^{\circ} \mathrm{C}$. (c) Photothermal stability curve of COL-Au-NPs $(10 \mu \mathrm{M})$ for five cycles of continuous ON/OFF laser irradiation (5 min each). (d) Concentration-dependent temperature change of COL-Au-NPs.

and MCF-7 breast cancer cells. Photodynamic inactivation of Gram-negative bacteria, E. coli, by nanoparticles was visualized on semisolid LB agar plates in the dark and under white light illumination $\left(22 \mathrm{~mW} / \mathrm{cm}^{2}, 3 \mathrm{~min}\right)$. To determine the optimum treatment concentrations for the nanoparticles, their MIC values were determined by following the broth dilution method. For this purpose, E. coli suspensions were incubated with the nanoparticles with a concentration range of 0.6-14.4 $\mu \mathrm{g} / \mathrm{mL}$ both in the dark and under light. As shown in Figures S9 and S10, the MIC was determined to be around $12 \mu \mathrm{g} / \mathrm{mL}$. On the basis of this MIC value, bacterial suspensions were treated with $12.4 \mu \mathrm{g} / \mathrm{mL}$ of nanoparticles both in the dark and under light exposure (Figure $3 \mathrm{a}-\mathrm{d}$ ). Colony counting results revealed around 3.5-log reduction in the colony-forming units (CFUs) of E. coli treated with $12.4 \mu \mathrm{g} / \mathrm{mL}$ of COL-Au-NPs under light exposure. However, in the dark, COL-Au-NPs also exhibited dark cytotoxicity (around 0.6-log reduction), which is not negligible. This is in line with our previous observations. ${ }^{34}$ To overcome this problem, the amine residues on the surfaces of the nanoparticles, which cause high dark toxicity were capped with $\mathrm{CB} 7$ as we did in our previous work. Dark cytotoxicity of CB7-decorated COL-Au-NPs was significantly reduced and CB7@COL-Au-NPs were found to be quite stable in aqueous solution, PBS, and Dulbecco's Modified Eagle's Medium (DMEM) without forming any visible aggregation. We have also investigated the effect of these nanoparticles on the Gram-positive (B. subtilis) bacteria and found similar results. To compare the interaction of nanoparticles with Gram-negative (E. coli) and Gram-positive (B. subtilis) bacteria, $\zeta$-potential measurements were performed. Both bacterial strains were treated with $12.4 \mu \mathrm{g} / \mathrm{mL}$ of nanoparticles, and their $\zeta$-potential values were measured (Figure 3f). While nontreated control groups of E. coli and $B$. subtilis had -46.4 and $-35.5 \mathrm{mV}$ of $\zeta$-potentials, respectively, upon treatment, the values shifted to more positive values indicating the interaction between nanoparticles and both bacterial strains (Figure 3f). High light-induced antimicrobial activity and a minimal dark toxicity of this photosensitizer, CB7@COL-Au-NPs, against both bacteria types imply its suitability to be used as a broad-spectrum antibacterial agent.

After bacterial survival experiments and $\zeta$-potential measurements, the effect of nanoparticles on $E$. coli was further studied by SEM. Morphology of $E$. coli before and after the treatment with nanoparticles both in the dark and under light was investigated. In Figure $3 \mathrm{~b}, \mathrm{~d}$, the corresponding SEM images are shown. When bacteria were treated with CB7@COL-AuNPs in the dark, nanoparticles were observed to be accumulated on the cell membranes, but bacteria appeared to preserve their rod-like structure and smooth surfaces without any noticeable damages (Figure $3 \mathrm{~b}$ ). On the other hand, the nanoparticle-treated and the light-exposed bacteria were completely destroyed in comparison to their lightexposed nontreatment control group. The images show that 
(a)

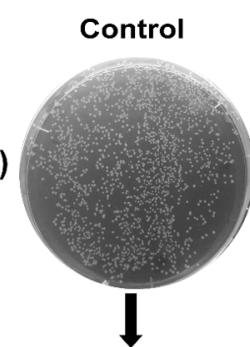

(b)
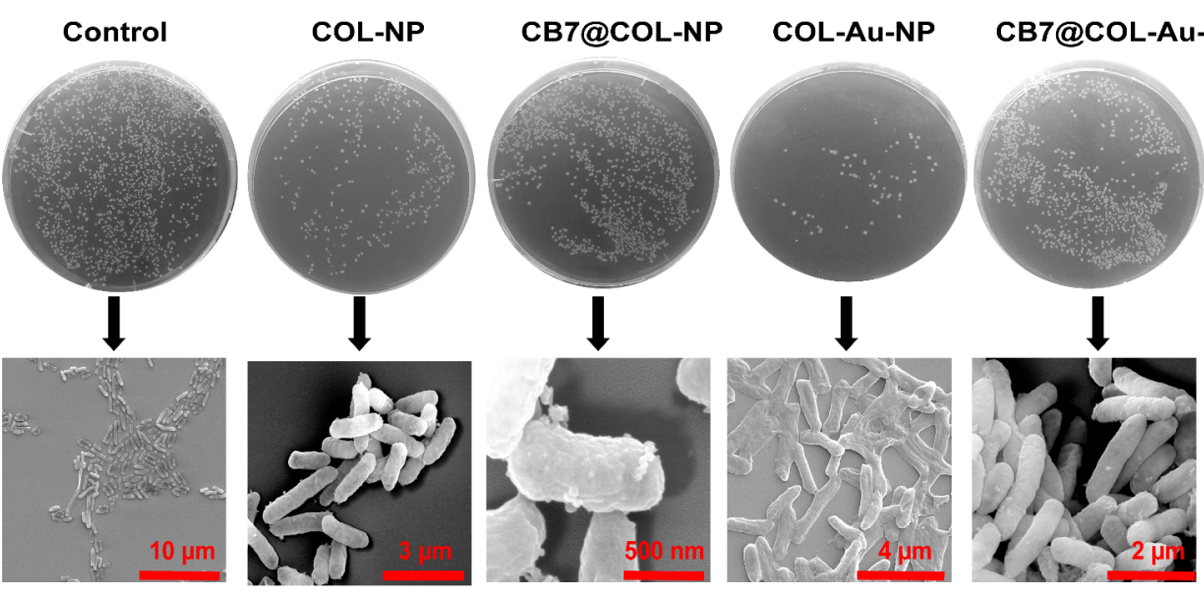

(e)

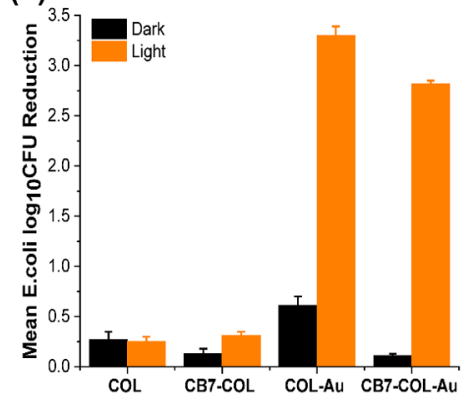

(c)

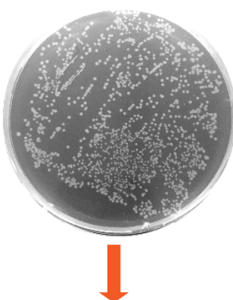

(d)

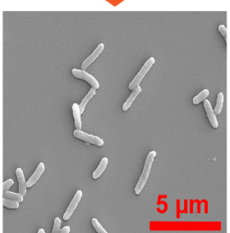

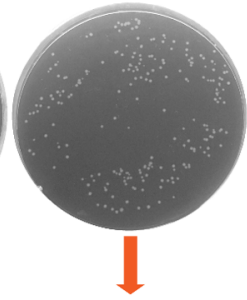

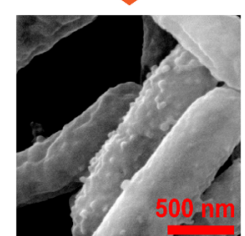

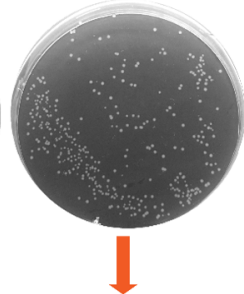

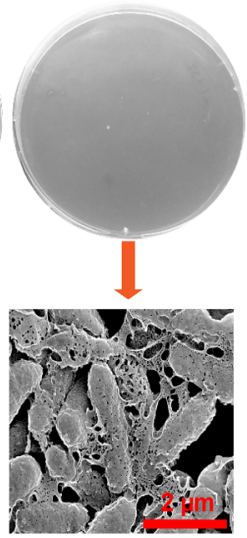

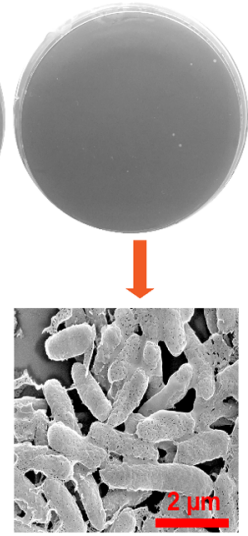

(f)

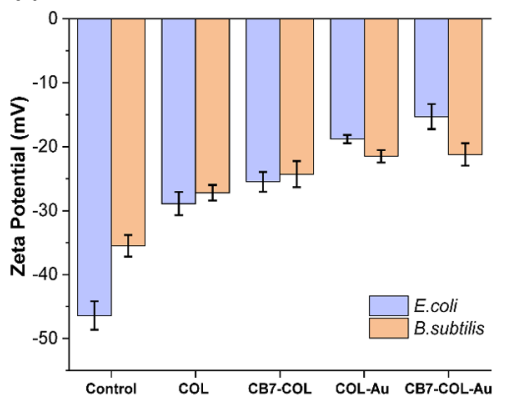

Figure 3. In the dark, treatment of $12.4 \mu \mathrm{g} / \mathrm{mL}$ of each nanoparticle group. (a) CFUs of E. coli on semisolid LB agar plates and (b) corresponding SEM images. Under white light exposure $\left(3 \mathrm{~min}, 22 \mathrm{~mW} / \mathrm{cm}^{2}\right)$, treatment of $12.4 \mu \mathrm{g} / \mathrm{mL}$ of each nanoparticle group. (c) CFUs of E. coli on semisolid LB agar plates and (d) corresponding SEM images. (e) Mean E. coli $\log _{10}$ CFU reduction upon treatment with $12.4 \mu \mathrm{g} / \mathrm{mL}$ of each nanoparticle in the dark and white light $\left(3 \mathrm{~min}, 22 \mathrm{~mW} / \mathrm{cm}^{2}\right)$, results are plotted as mean $\pm \mathrm{SD}(n=3)$. (f) $\zeta$-Potential changes of $E$. coli and $B$. subtilis upon the treatment of each nanoparticle, results are plotted as mean $\pm \operatorname{SD}(n=3)$.

they had ruptured membranes with leaked out cytoplasmic organelles (Figure 3d).

3.4. Cytotoxicity and Photodynamic Effect of Nanoparticles on Cancer Cells. Cytotoxicity and photodynamic properties of nanoparticles were investigated in vitro through methyl thiazolyl tetrazolium (MTT) assay using MCF-7 breast cancer cells. As can be seen from Figure 4a, although COLNPs and COL-Au-NPs cause a significant decrease in the viability of MCF-7 cells even in the dark, their dark toxicities can be switched off by capping with CB7. Indeed, the assays confirmed that CB7@COL-NPs and CB7@COL-Au-NPs caused a negligible dark toxicity even at high concentrations. Upon light irradiation (white light, $20 \mathrm{~min}, 20 \mathrm{~mW} / \mathrm{cm}^{2}$ ), all of those nanoparticles exhibit a dose-dependent cytotoxicity against the MCF-7 cells; however, hybrid nanoparticles (COL-Au-NPs and CB7@COL-Au-NPs) appeared to have significantly higher phototoxicity than COL-NPs and CB7@ COL-NPs (Figure 4b). These results are in good agreement with the antimicrobial assays suggesting that CB7@COL-AuNPs are an efficient photosensitizer with minimal dark cytotoxicity for antimicrobial and anticancer PDT. Moreover, the dark cytotoxicity can be conveniently switched on and off at will because of the presence of CB7s.

As nanoparticles are inherently fluorescent, they could also be utilized directly in cellular imaging applications or imageguided photodynamic cancer therapy. To verify whether nanoparticles could be internalized by MCF-7 cells or not, nanoparticle-incubated and nonincubated DMEM control cells were examined by confocal laser scanning microscopy (CLSM) as shown in Figure $4 \mathrm{c}-\mathrm{j}$. They were treated with $1 \mu \mathrm{M}$ COLAu-NPs and CB7@COL-Au-NPs for $72 \mathrm{~h}$ (for the light group, after $48 \mathrm{~h}$, cells were exposed to white light for $20 \mathrm{~min}$, the same in MTT assays). Their nuclei were stained by DAPI emitting blue fluorescence under the excitation of the $461 \mathrm{~nm}$ laser, while COL-Au-NPs emitted red fluorescence upon excitation by a $580 \mathrm{~nm}$ laser. As presented in Figure $4 \mathrm{e}-\mathrm{j}$, merged pictures confirm that the cellular uptake of COL-AuNPs was obvious especially near the nuclear membrane. To observe the change of the cellular phenotype after nanoparticle treatment, differential interference contrast microscopy (DIC) images were also captured. In Figure $4 \mathrm{c}, \mathrm{g}$, DIC images of the nontreated control DMEM group in the dark and under light illumination are given. As can be seen, highly adherent control cells have cobblestone-like morphology, and cells retained their edged structure after light exposure. However, upon COL-AuNP addition, even in the dark, morphological changes were quite obvious. Cells lost the sided-membrane shape and were found to be circular with dark inner contents (Figure 4i). Similar morphological modifications were also observed for CB7@COL-Au-NPs that cause the disturbance of cell appearances. (Figure S12). 


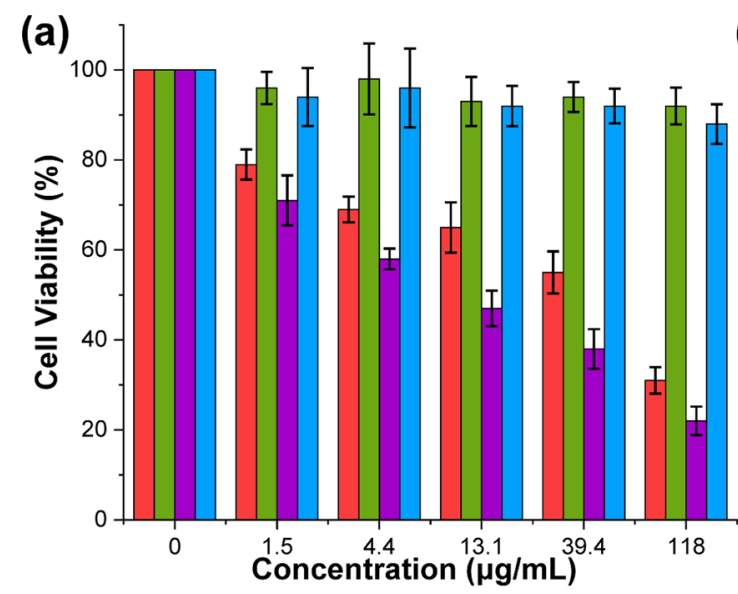

(b)
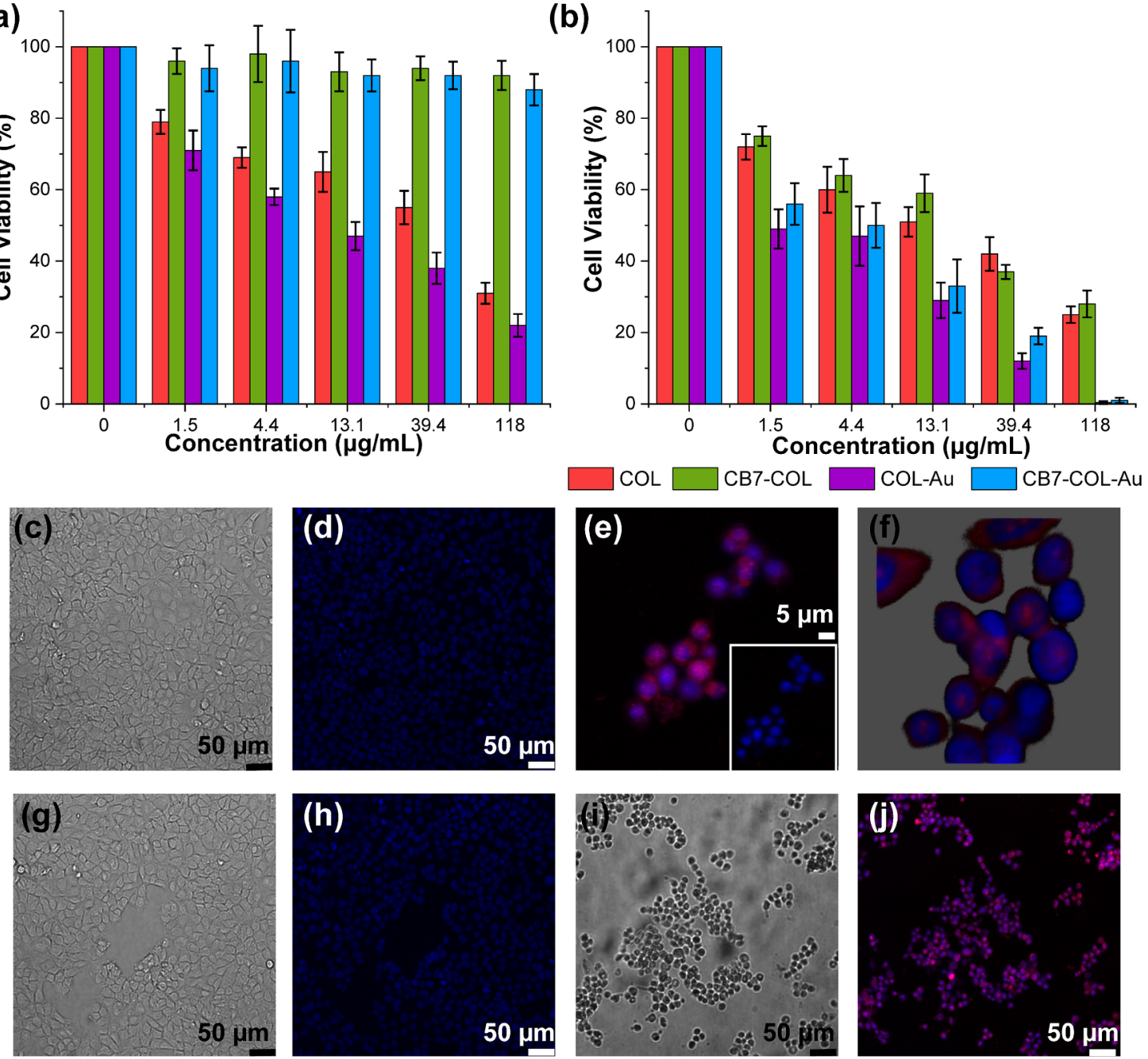

Figure 4. Relative cell viability (\%) of MCF-7 cells treated with various concentrations of COL-NPs, CB7@COL-NPs, COL-Au-NPs, and CB7@ COL-Au-NPs: (a) in the dark and (b) under continuous white light irradiation $\left(20 \mathrm{~min}, 20 \mathrm{~mW} / \mathrm{cm}^{2}\right)$. MCF-7 cells of the nontreated DMEM control group in the dark. (c) DIC image and (d) CLSM image, and nuclei were stained with DAPI. (e) MCF-7 cells were treated with $1 \mu$ M COLAu-NPs and exposed to light. (f) Three-dimensional image of COL-Au-NP-treated MCF-7 cells. (g) DIC image of the nontreated light control group. (h) CLSM image of the nontreated light control group. (i) DIC image of the COL-Au-NP-treated dark group. (j) CLSM image of the COL$\mathrm{Au}-\mathrm{NP}$-treated dark group.

\subsection{Combined PTT/PDT Effect of Nanoparticles on}

Bacteria. As a next step, after verification of photothermal and photodynamic properties of nanoparticles, we intended to combine PDT and PTT and test the combined antibacterial therapy efficiency on E. coli and B. subtilis by agar disk diffusion assay. Both bacterial strains were treated with $12.4 \mu \mathrm{g} / \mathrm{mL}$ (MIC) of COL-NP, CB7@COL-NP, COL-Au-NPs, and CB7@COL-Au-NP and a reference antibiotic, ampicillin, to compare their performance with one another. As shown in Figure 5a,b, treatments were repeated in the dark, only under white light exposure for $3 \mathrm{~min}$ to evaluate PDT efficiency, only under $915 \mathrm{~nm}$ laser irradiation for $3 \mathrm{~min}$ to evaluate PTT efficiency, and under $3 \mathrm{~min}$ white light illumination followed by $3 \mathrm{~min} 915 \mathrm{~nm}$ laser irradiation to observe combined PDT/ PTT efficiency. Inhibition zones were determined by measuring the radii of cell-free zones using similar concentration for all samples. As expected, the inhibition radius of ampicillin was around $3 \mathrm{~mm}$ for all conditions. In the dark, only the effect of COL-Au-NPs was visible for both types. PDT efficiency of COL-Au-NPs was significantly higher than that of PTT. When PDT and PTT were combined, broader inhibition zones were achieved for all nanoparticles (Figure 5c,d). Especially for CB7@COL-Au-NPs, inhibition activities against Gram-positive and Gram-negative bacteria were dramatically improved. These findings suggest that combined PDT and PTT treatment could achieve an enhanced antimicrobial therapeutic efficacy beyond individual therapy of PDT or PTT.

\section{CONCLUSIONS}

In this work, we have developed novel hybrid nanoparticles based on the COL containing gold (COL-Au). This multifunctional hybrid system possesses high photostability and thermal reversibility, achieving combined photodynamic (PDT)-photothermal (PTT) therapies and cellular imaging. Combining PDT and PTT in a single platform could afford an efficacious broad-spectrum antibacterial treatment. Moreover, COL-Au nanoparticles were capped by cucurbit[7]uril to eliminate dark cytotoxicity toward mammalian breast cancer cells and pathogens. 

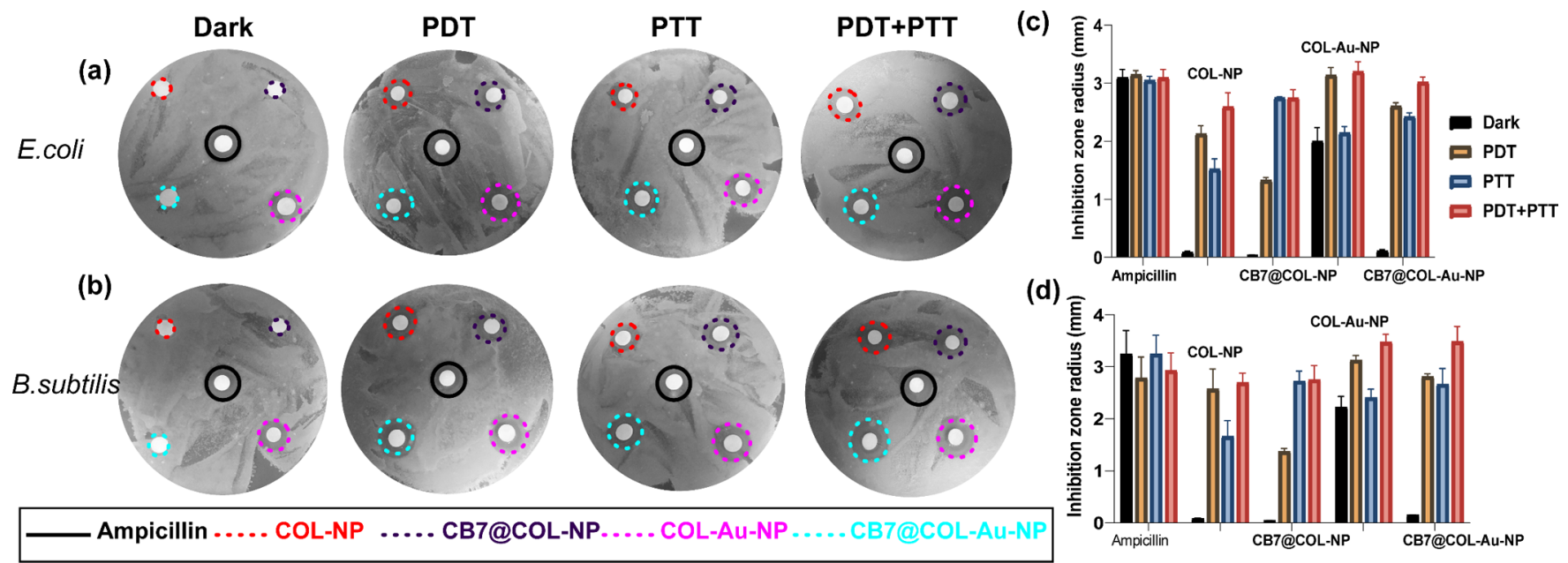

Figure 5. Plate photographs of agar disk diffusion assays of (a) E. coli and (b) B. subtilis, which were treated with $12.4 \mu \mathrm{g} / \mathrm{mL}$ of ampicillin, COLNPs, CB7@COL-NPs, COL-Au-NPs, and CB7@COL-Au-NPs in the dark, under 3 min white light illumination (PDT), under 3 min 915 nm laser irradiation (PTT), and under $3 \mathrm{~min}$ white light illumination and afterward $3 \mathrm{~min} 915 \mathrm{~nm}$ laser irradiation (PDT/PTT). Inhibition zone graphs of (c) E. coli and (d) B. subtilis. The inhibition zone is the mean of the radii measured from at least six different points of the circle. Experiments were repeated in triplicate, and results were plotted as the mean of three independent experiments.

\section{ASSOCIATED CONTENT}

\section{(s) Supporting Information}

The Supporting Information is available free of charge at https://pubs.acs.org/doi/10.1021/acsapm.0c00540.

Details of the experimental section, quantification of photothermal conversion efficiency of nanoparticles, DLS histograms of COL-NP, CB7@COL-NP, COLAu-NPs, and CB7@COL-Au-NP, PDI and zeta potential measurements, fluorescence spectra, UV-vis-NIR spectra, TRF measurements, XRD pattern, XPS, PL spectra of DCF at $524 \mathrm{~nm}$ in the presence of COL-NPs and COL-Au-NPs under continuous white-light illumination, photothermal response of COL-Au-NPs at various laser densities, MIC assays, concentrations of nanoparticles (COL, CB7@COL, COL-Au, and CB7@ COL-Au) used in MTT cell viability assays, and more CLSM images (PDF)

\section{AUTHOR INFORMATION}

\section{Corresponding Author}

Dönüs Tuncel - Institute of Materials Science and Nanotechnology, National Nanotechnology Research Center (UNAM) and Department of Chemistry, Bilkent University, Ankara 06800, Turkey; 이이. orcidorg/0000-0001-7762-9200; Email: dtuncel@fen.bilkent.edu.tr

\section{Authors}

Melis Özkan - Institute of Materials Science and Nanotechnology, National Nanotechnology Research Center (UNAM), Bilkent University, Ankara 06800, Turkey

Seyed Ehsan Hadi - Institute of Materials Science and Nanotechnology, National Nanotechnology Research Center (UNAM), Bilkent University, Ankara 06800, Turkey

İlknur Tunç - Department of Mechanical Engineering, University of Turkish Aeronautical Association, Ankara 06797, Turkey

Yakup Midilli - Institute of Materials Science and Nanotechnology, National Nanotechnology Research Center
(UNAM), Bilkent University, Ankara 06800, Turkey; (ㅇ) orcid.org/0000-0003-1509-1235

Bülend Ortaç - Institute of Materials Science and Nanotechnology, National Nanotechnology Research Center (UNAM), Bilkent University, Ankara 06800, Turkey

Complete contact information is available at: https://pubs.acs.org/10.1021/acsapm.0c00540

\section{Author Contributions}

D.T. conceived the idea, supervised, and coordinated the project. D.T. and M.O. designed the experiments. S.E.H. and M.O. carried out chemical characterization. S.E.H measured ROS generation capacity. M.O. synthesized nanoparticles and performed bacterial experiments, MTT assays, and CLSM imaging. I.T. performed XPS analysis. Y.M. and B.O. provided the facility and contributed to photothermal property investigation experiments. D.T. and M.O. analyzed the data and wrote the manuscript. All authors have given approval to the final version of the manuscript. There authors declare no competing financial interest.

\section{Notes}

The authors declare no competing financial interest.

\section{ACKNOWLEDGMENTS}

The authors acknowledge the financial support of the Scientific and Technological Research Council of Turkey-TÜBITAK (Katip Çelebi-Newton, Institutional Link, KBAG 216Z123).

\section{REFERENCES}

(1) Xue, X.; Wang, F.; Liu, X. Emerging Functional Nanomaterials for Therapeutics. J. Mater. Chem. 2011, 21, 13107-13127.

(2) Zhou, L.; Qiu, T.; Lv, F.; Liu, L.; Ying, J.; Wang, S. SelfAssembled Nanomedicines for Anticancer and Antibacterial Applications. Adv. Healthcare Mater. 2018, 7, 1800670.

(3) Gupta, A.; Mumtaz, S.; Li, C. H.; Hussain, I.; Rotello, V. M. Combatting Antibiotic-Resistant Bacteria Using Nanomaterials. Chem. Soc. Rev. 2019, 48, 415-427.

(4) Li, X.; Bai, H.; Yang, Y.; Yoon, J.; Wang, S.; Zhang, X. Supramolecular Antibacterial Materials for Combatting Antibiotic Resistance. Adv. Mater. 2019, 31, 1805092. 
(5) Phillips, D. Light Relief: Photochemistry and Medicine. Photochem. Photobiol. Sci. 2010, 9, 1589.

(6) Maisch, T. Resistance in Antimicrobial Photodynamic Inactivation of Bacteria. Photochem. Photobiol. Sci. 2015, 14, 1518.

(7) Celli, J. P.; Spring, B. Q.; Rizvi, I.; Evans, C. L.; Samkoe, K. S.; Verma, S.; Pogue, B. W.; Hasan, T. Imaging and Photodynamic Therapy: Mechanisms, Monitoring, and Optimization. Chem. Rev. 2010, 110, 2795-2838.

(8) Zhou, Z.; Song, J.; Nie, L.; Chen, X. Reactive Oxygen Species Generating Systems Meeting Challenges of Photodynamic Cancer Therapy. Chem. Soc. Rev. 2016, 45, 6597.

(9) Agostinis, P.; Berg, K.; Cengel, K. A.; Foster, T. H.; Girotti, A. W.; Gollnick, S. O.; Hahn, S. M.; Hamblin, M. R.; Juzeniene, A.; Kessel, D.; Korbelik, M.; Moan, J.; Mroz, P.; Nowis, D.; Piette, J.; Wilson, B. C.; Golab, J. Photodynamic Therapy of Cancer: An Update. Ca-Cancer J. Clin. 2011, 61, 250-281.

(10) Wilson, B. C.; Patterson, M. S. The Physics, Biophysics and Technology of Photodynamic Therapy. Phys. Med. Biol 2008, 53, 61.

(11) Dolmans, D. E.; Fukumura, D.; Jain, R. K. Photodynamic Therapy for Cancer. Nat. Rev. Cancer 2003, 3, 380-387.

(12) Dharmaraja, A. T. Role of Reactive Oxygen Species (ROS) in Therapeutics and Drug Resistance in Cancer and Bacteria. J. Med. Chem. 2017, 60, 3221-3240.

(13) Jaque, D.; Martínez Maestro, L.; del Rosal, B.; Haro-Gonzalez, P.; Benayas, A.; Plaza, J. L.; Martín Rodríguez, E.; García Solé, J. Nanoparticles for Photothermal Therapies. Nanoscale 2014, 6, 94949530.

(14) Gao, D.; Guo, X.; Zhang, X.; Chen, S.; Wang, Y.; Chen, T.; Huang, G.; Gao, Y.; Tian, Z.; Yang, Z. Multifunctional Phototheranostic Nanomedicine for Cancer Imaging and Treatment. Mater. Today Bio. 2019, 5, 100035.

(15) Wei, X.; Chen, H.; Tham, H. P.; Zhang, N.; Xing, P.; Zhang, G.; Zhao, Y. Combined Photodynamic and Photothermal Therapy Using CrossLinked Polyphosphazene Nanospheres Decorated with Gold Nanoparticles. ACS Appl. Nano Mater. 2018, 1, 3663-3672.

(16) Jiang, Y.; Zhao, X.; Huang, J.; Li, J.; Upputuri, P. K.; Sun, H.; Han, X.; Pramanik, M.; Miao, Y.; Duan, H.; Pu, K.; Zhang, R. Transformable Hybrid Semiconducting Polymer Nanozyme for Second Near-Infrared Photothermal Ferrotherapy. Nat. Commun. 2020, 11, 1857.

(17) Li, J.; Pu, K. Development of Organic Semiconducting Materials for Deep-Tissue Optical Imaging, Phototherapy and Photoactivation. Chem. Soc. Rev. 2019, 48, 38-71.

(18) Jiang, Y.; Pu, K. Multimodal Biophotonics of Semiconducting Polymer Nanoparticles. Acc. Chem. Res 2018, 51, 1840-1849.

(19) Cui, D.; Huang, J.; Zhen, X.; Li, J.; Jiang, Y.; Pu, K. A Semiconducting Polymer Nano-Prodrug for Hypoxia-Activated Photodynamic Cancer Therapy. Angew. Chem., Int. Ed 2019, 58, 5920-5924.

(20) Ray, P. C.; Khan, S. A.; Singh, A. K.; Senapati, D.; Fan, Z. Nanomaterials for Targeted Detection and Photothermal Killing of Bacteria. Chem. Soc. Rev. 2012, 41, 3193-3209.

(21) Chen, H.; Di, Y.; Chen, D.; Madrid, K.; Zhang, M.; Tian, C.; Tang, L.; Gu, Y. Combined Chemo-and Photo-thermal Therapy Delivered by Multifunctional Theranostic Gold Nanorod-loaded Microcapsules. Nanoscale 2015, 7, 8884-8897.

(22) Yu, Z.-H.; Li, X.; Xu, F.; Hu, X.-L.; Yan, J.; Kwon, N.; Chen, G.R.; Tang, T.; Dong, X.; Mai, Y.; Chen, D.; Yoon, J.; He, X.-P.; Tian, H. A Supramolecular-Based Dual-Wavelength Phototherapeutic Agent with Broad-Spectrum Antimicrobial Activity Against DrugResistant Bacteria. Angew. Chem., Int. Ed. 2020, 59, 3658-3664.

(23) Zhang, H.; Li, Y.-H.; Chen, Y.; Wang, M.-M.; Wang, X.-S.; Yin, $\mathrm{X}$.-B. Fluorescence and Magnetic Resonance Dual-Modality ImagingGuided Photothermal and Photodynamic Dual-Therapy with Magnetic Porphyrin-Metal Organic Framework Nanocomposites. Sci. Rep. 2017, 7, 44153.

(24) Wang, B.; Feng, G.; Seifrid, M.; Wang, M.; Liu, B.; Bazan, G. C. Antibacterial Narrow-Band-Gap Conjugated Oligoelectrolytes with
High Photothermal Conversion Efficiency. Angew. Chem., Int. Ed. 2017, 56, 16063-16066.

(25) Li, W.; Zhang, H.-B.; Guo, X.-M.; Wang, Z.-H.; Kong, F.-F.; Luo, L.-H.; Li, Q.-P.; Zhu, C.-Q.; Yang, J.; Lou, Y.; Du, Y.-Z.; You, J. Gold Nanospheres-Stabilized Indocyanine Green as a Synchronous Photodynamic-Photothermal Therapy Platform That Inhibits Tumor Growth and Metastasis. ACS Appl. Mater. Interfaces 2017, 9, 33543367.

(26) Tuncel, D.; Demir, H. V. Conjugated Polymer Nanoparticles. Nanoscale 2010, 2, 484-494.

(27) Tuncel, D. $\pi$-Conjugated Nanostructured Materials: Preparation, Properties and Photonic Applications. Nanoscale Adv 2019, 1, 19-33.

(28) Feng, G.; Fang, Y.; Liu, J.; Geng, J.; Ding, D.; Liu, B. Multifunctional Conjugated Polymer Nanoparticles for Image-Guided Photodynamic and Photothermal Therapy. Small 2017, 13, 1602807.

(29) Wang, Y.; Li, S.; Liu, L.; Feng, L. Photothermal-Responsive Conjugated Polymer Nanoparticles for the Rapid and Effective Killing of Bacteria. ACS Appl. Bio Mater. 2018, 1, 27-32.

(30) Park, D. H.; Kim, M. S.; Joo, J. Hybrid Nanostructures Using $\pi$ Conjugated Polymers and Nanoscale Metals: Synthesis, Characteristics, and Optoelectronic Applications. Chem. Soc. Rev. 2010, 39, 2439-2452.

(31) Wang, X.; He, F.; Zhu, X.; Tang, F.; Li, L. Hybrid Silver Nanoparticle/Conjugated Polyelectrolyte Nanocomposites Exhibiting Controllable Metal-Enhanced Fluorescence. Sci. Rep. 2014, 4, 4406.

(32) Ki, M. S.; Park, D. H.; Cho, E. H.; Kim, K. H.; Park, Q.-H.; Song, H.; Kim, D.-C.; Kim, J.; Joo, J. Complex Nanoparticle of LightEmitting MEH-PPV with Au: Enhanced Luminescence. ACS Nano 2009, 3, 1329.

(33) Balkan, T.; Kizir, S.; Tuncel, D. One-Pot Synthesis of Hybrid Conjugated Oligomer-Ag Nanoparticles. ACS Omega 2017, 2, 54705477.

(34) Pennakalathil, J.; Jahja, E.; Özdemir, E. S.; Konu, Ö.; Tuncel, D. Red Emitting, Cucurbituril-Capped, pH-Responsive Conjugated Oligomer-Based Nanoparticles for Drug Delivery and Cellular Imaging. Biomacromolecules 2014, 15, 3366-3374.

(35) Gürbüz, S.; Idris, M.; Tuncel, D. Cucurbituril-Based Supramolecular Engineered Nanostructured Materials. Org. Biomol. Chem. 2015, 13, 330-347.

(36) Koc, A.; Tuncel, D. Supramolecular Assemblies of Cucurbiturils with Photoactive, $\pi$-conjugated Chromophores. Isr J. Chem. 2018, 58, 334.

(37) Zhang, X.; Xu, X.; Li, S.; Li, L.; Zhang, J.; Wang, R. A Synthetic Receptor as a Specific Antidote for Paraquat Poisoning. Theranostics 2019, 9, 633-645.

(38) Yue, L.; Sun, C.; Cheng, Q.; Ding, Y.; Wei, J.; Wang, R. Gold Nanorods with a Noncovalently Tailorable Surface for Multi-Modality Image-Guided Chemo-Photothermal Cancer Therapy. Chem. Commun. 2019, 55, 13506-13509.

(39) Huang, Q.; Kuok, K. I.; Zhang, X.; Yue, L.; Lee, S. M. Y.; Zhang, J.; Wang, R. Inhibition of Drug-Induced Seizure Development in Both Zebrafish and Mouse Models by a Synthetic Nanoreceptor. Nanoscale 2018, 10, 10333-10336.

(40) Xu, P.; Feng, Q.; Yang, X.; Liu, S.; Xu, C.; Huang, L.; Chen, M.; Liang, F.; Cheng, Y. Near Infrared Light Triggered Cucurbit[7]urilStabilized Gold Nanostars as a Supramolecular Nanoplatform for Combination Treatment of Cancer. Bioconjugate Chem. 2018, 29, $2855-2866$.

(41) Soran-Erdem, Z.; Erdem, T.; Gungor, K.; Pennakalathil, J.; Tuncel, D.; Demir, H. V. High-Stability, High-Efficiency Organic Monoliths Made of Oligomer Nanoparticles Wrapped in Organic Matrix. ACS Nano 2016, 10, 5333-5339.

(42) Özkan, M.; Keser, Y.; Hadi, S. E.; Tuncel, D. A [5]RotaxaneBased Photosensitizer for Photodynamic Therapy. Eur. J. Org. Chem. 2019, 2019, 3534-3541.

(43) Özkan, M.; Kumar, Y.; Keser, Y.; Hadi, S. E.; Tuncel, D. Cucurbit[7]uril-Anchored Porphyrin-Based Multifunctional Molec- 
ular Platform for Photodynamic Antimicrobial and Cancer Therapy. ACS Appl. Bio Mater. 2019, 2, 4693-4697.

(44) Ozkan, M.; Keser, Y.; Koc, A.; Tuncel, D. Glycosylated Porphyrin-Cucurbituril Conjugate for Photodynamic Inactivation of Bacteria and Doxorubicin Carriage for Anticancer Drug Delivery. J. Porphyrins Phthalocyanines 2019, 23, 1406-1413.

(45) Khan, R.; Özkan, M.; Khaligh, A.; Tuncel, D. Water-Dispersible Glycosylated Poly (2,5'-thienylene) Porphyrin-Based Nanoparticles for Antibacterial Photodynamic Therapy. Photochem. Photobiol. Sci. 2019, 18, 1147-1155.

(46) Chen, L.; Bai, H.; Xu, J. F.; Wang, S.; Zhang, X. Supramolecular Porphyrin Photosensitizers: Controllable Disguise and Photoinduced Activation of Antibacterial Behavior. ACS Appl.Mater. Interfaces 2017, 9, 13950-13957.

(47) Macia, N.; Bresoli-Obach, R.; Nonell, S.; Heyne, B. Hybrid Silver Nanocubes for Improved Plasmon-Enhanced Singlet Oxygen Production and Inactivation of Bacteria. J. Am. Chem. Soc. 2019, 141, 684-692.

(48) Tian, Q.; Jiang, F.; Zou, R.; Liu, Q.; Chen, Z.; Zhu, M.; Yang, S.; Wang, J.; Wang, J.; Hu, J. Hydrophilic Cu 9S 5 Nanocrystals: A Photothermal Agent with a 25.7\% Heat Conversion Efficiency for Photothermal Ablation of Cancer Cells in Vivo. ACS Nano 2011, 5, 9761-9771. 